\title{
Parasitic Radio: combining FM radio signals with environmental sensor data via computing techniques for soundscape creation.
}

\author{
Juan Carlos Duarte Regino \\ Aalto University
}

Juan.durat@aalto.fi

\begin{abstract}
Environmental interface to mediate electromagnetic signals and sensor data
\end{abstract}
FM Radio Signals, Radio Art, Environmental Data

\section{INTRODUCTION}

This paper exposes the process of development for Parasitic Radio as a workshop featured in the conference: Politics of Machines 2019. The, origin of this project comes from an ongoing experimentation with $\mathrm{FM}$ and $\mathrm{AM}$ radio, understood as a medium for telematic artwork in the context of my current doctoral research in Environmental Media, which concentrates on the study of the relation between media technology and planetary signals.

The focus of my doctoral research is to study how to produce environmental art that explores the relevance of technology into an ecological discourse: The artistic research put here suggests the development of critical media technologies to reflect on the notions of augmented listening, telematic art, and ecologies of transmission, as study cases where the limits in between can be described as liminal: organic and artificial matter, immediate and distant (telematic) performance, and tangible and intangible, planetary and human agencies. In the case of Parasitic Radio I aim to create a medium of communication where environmental signals can be computed into radio signals that return into the air as a way to make perceivable by workshop participants the potential exchanges between mediums (air and electromagnetic fields), in other words to make visible and or audible phenomena that otherwise is hidden to human perception.

For Parasitic Radio I use design methods from New Media studies in order to produce environmental media artwork: interface design, user experience, data visualization and sonic interaction. These methods can be used to design exhibitions, and participatory performances and workshops, with and through environmental agencies (energies, materials, signals).

The idea of integrating environmental and radio signals through computing techniques intends to explore, as it will be reviewed along the examples presented in this paper, separate media experiments found in sound art histories, and technology developments from the 20th century. These examples lead to a tradition of mediations between audiovisual art and media technology which coexisted to open up to creative appropriations between environmental listening and composing mediations made through technology.

Parasitic Radio was presented in Politics of Machines - Beirut as a workshop for participants to get an overview about micro powered radio broadcasting, sensing technologies, and computing techniques to create audiovisual representations about these medium exchanges. Besides, the aim is to have a workshop is to study the potential relations between sound art, environmental awareness, and computational agents to compose soundscapes from bundles of data and radio signals. Lastly, the workshop participants are engaged in a sort of situationist "derivé" radio intervention. This situationist radio intervention is possible through media devices that interact with local radio content that modulates or interferes with the resulting sound piece. 


\section{LOW-POWERED RADIO}

FM and AM Radio, comparable to digital internet streaming technologies, depend on a lower material infrastructure ${ }^{1}$. Radio stations in this spectrum are available in a variety of institutional configurations ranging from high to low powered (private, public, community, etc). Besides, contrary to the 'narrowcasting' quality of internet radio, FM and AM Radio transmissions are found in a spectrum of 'noise against signal' where static in between broadcasting is easy to perceive as when offsetting the turn of an old radio's frequency dial. The ease of radio as a technology that is 'already there', as John Cage would describe as: "an instrument that opens up listening to sounds in which we are already submerged into". Following this impetus, the Parasitic Radio workshop explores radio micro transmissions as a way to exploit the existing radio infrastructure (frequency bands, radio contents, and atmospheric noise) as environmental agencies for generative soundscape composition.

\section{RADIO MUSIC}

Parasitic Radio, initially takes as a reference John Cage's piece "Radio Music" (1956) as a music composition based on a semi deterministic ${ }^{2}$ model piece where eight performers who lead a soundscape piece, sources radio contents that are being transmitted during the performance. The performance unfolds over four parts having the performers to tune, and change volume amplification over a period of six minutes in total. Despite this agency, any performer decides how long to stay in a fixed position, as this is not declared by the composer, performer can decide discreetly on this timing. The sonic collage and collaborative qualities of this composition gives the possibility to create music with a low level of musical skills expertise. In comparison to most of the musical instruments that require a higher expertise to control, the radio as a musical instrument offers a low level entry, by accessing directly high level parameters of sound through electronic components: volume, frequency tuning and on or off.

As in "Radio Music", Parasitic Radio sources existing radio signals from local stations (in this

1 To run a radio reception and transmission it takes to buy few affordable electronic components, to run a internet based streaming it involves at least a server and broadband connection to make it effective.

2 Lindsay Vickery, proposes the term "Available Indeterminacy" to describe the framework used by John Cage in Radio Music. case broadcasting signals from Beirut, Lebanon). But instead of being controllable directly by players, the radio parameters are autonomously operated depending on surrounding weather conditions, such as light, temperature and sound levels. The autonomous computational system that controls radio parameters takes the decisions about when to change the radio station, or the volume, based on a data base collected from environmental input that generates a soundscape from radio signals.

\section{NATURAL RADIO}

Natural Radio, as a media history experiment described by Douglas Kahn (2013), serves as an inspiration for Parasitic Radio. The discovery of the radio medium comes along from combining two recent technologies at the beginning of the $20^{\text {th }}$ century: the wired telephone and the telegraph. This experiment aimed to create a wireless communication system: as a non intended result, Thomas Watson, assistant of Graham Bell, listened to the sound of atmospherics (whistlers or sprites) as a new signals propagated in the upper atmosphere. This experience, provoked a new imagery of sonic materials originated from planetary energies. Later on, the sound of atmospherics were introduced in contemporary sound art practices ${ }^{3}$ to offer potential mediations between energy transductions into music. In this sense, the notion of Natural Radio is interesting to Parasitic Radio in order to make audible environmental signals: electromagnetic fields, solar storms or northern lights activity, as sound materials available for artistic composition.

The "Aeolian" is an element of Environmental Poiesis (Morton, 2009), used in one of my earlier projects: Aeolian Artefacts (Duarte Regino, 2019), which relates to Kahn's notion of the Aelectrosonic: as new medium (exemplified with the Telegraphic Lines as an accidental sound instrumentinstallation) that registers transductions between wind forces and electromagnetism in our planet. The correlation between different energies found in the environment blend into a sound composition that enables a listening of surrounding transformations.

More recently, Martin Howse's Radio Mycelium ${ }^{4}$ (2018-2019) brings both an artwork and workshop by presenting a system of interconnected mediums: Mycelium networks are connected to sensory technology to manipulate micro powered radio transmissions. The project as a workshop, aimed to build the transmission devices and a medium to

3 Cfr. Alvin Lucier's piece "Sferics", and Joyce Hinterding's Aeriology.

4 http://www.1010.co.uk/org/radiomycelium.html 
grow the mycelium, ${ }^{5}$ but also as an installation to listen to the complex interconnectedness of the mycelium listening to radio signals and feeding them back again. Howse's describes his interest on exploring a sort of Electromagnetic Animism intertwined with the rhizomatic grow and signal conducting patterns of the mycelium.

\section{COMPUTING RADIO-SCAPES}

The devices used in Parasitic Radio workshop are designed to be portable to use in diverse types of setups. Hence, micro powered transmissions can be done discreetly, allowing participants to play without concern to misuse or break the electronic components. Concentrating the experience on the sonic interaction between the radio systems and environmental agencies: light, sound, proximity of antennas of radio receivers and transmitters.

The technical setup consisted on a series of modules interconnected as an ensemble of functions to compute individual tasks: radio receiving, environmental sensing, data logging, and radio transmission of the resulting soundscape. A layer of data sonification was added for educational purposes of the workshop, then participants could understand how sensor data modifies sound parameters based on environmental conditions. In such case the data was modifying parameters from sound effects added to the radio collage created.

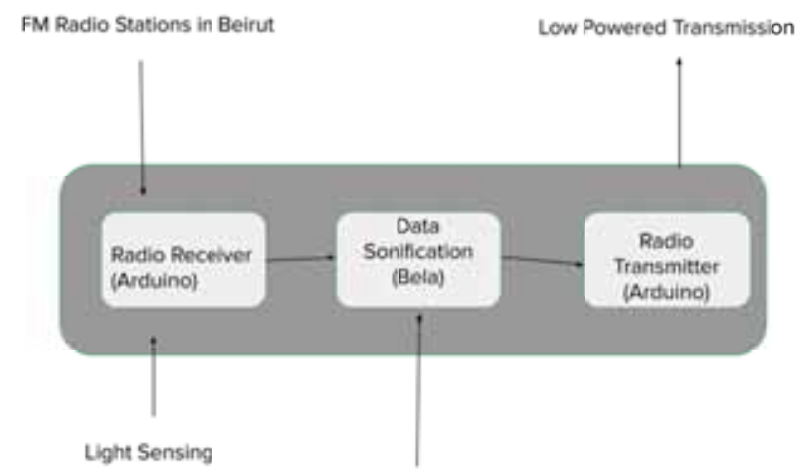

Sound, Temp Sensors

Figure 1: Diagram of computing modules.

Moreover, as seen in Figure 1, at least two of the modules: the radio receiver and data sonification use sensor input. This input modifies separate parameters of the piece and the third one from the right, labelled as "radio transmitter" is transmitting on a fixed frequency, in order to enable ease the reception using regular radios. Figure 2 shows a first prototype of the device produced prior the

5 https://fo.am/events/radio mycelium/ workshop, which was made during the Augmented Attention $\mathrm{Lab}^{6}$

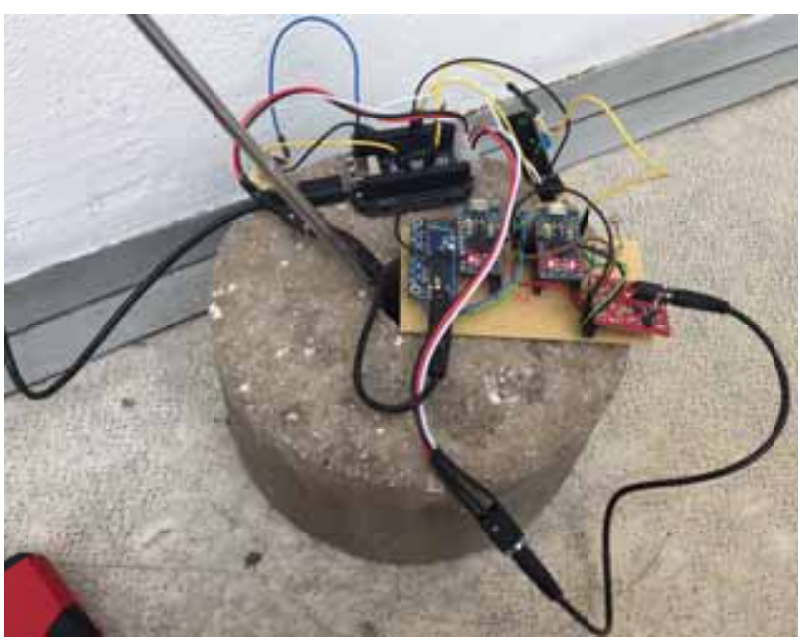

Figure 2: Working Prototype Circuit.

Produced during "Augmented Attention Lab" June 2019, Bratislava, Slovakia.

\section{CONCLUSIONS}

The workshop of Parasitic Radio, opened up the possibility to practice ideas that I collected during my research on the history of radio and its relation with new media art. First, the approach presented in Cage's "Radio Music" provides an artistic framework for composition: repurposing radio technology as a musical instrument, and opening listening to an environmental listening. Without leading elements, where human voice and music, sound effects and even radio interference have a same value at play, to create an immersive experience.

The idea of Natural Radio enables an experimentation with atmospheric sounds, carrying information and communication between non living agents, and environmental agencies. As in the case of Howse's "Radio Mycelium", the interest of experimenting with the radio medium is on playing around the complex interrelatedness and signal modulations. The speculative value of this artwork brings the possibility to think about possible interspecies communication medium through material and ethereal environments.

The workshop of Parasitic Radio presents these artworks as a background, to bring a context and framework to experiment with micro powered transmissions and environmental computing techniques (sonification and radio collage). On further editions of this workshop it would be useful to create visualizations from data logged in specific

6 augmented-attention-lab/ 
places and see the correlation with radio content received and transmitted.

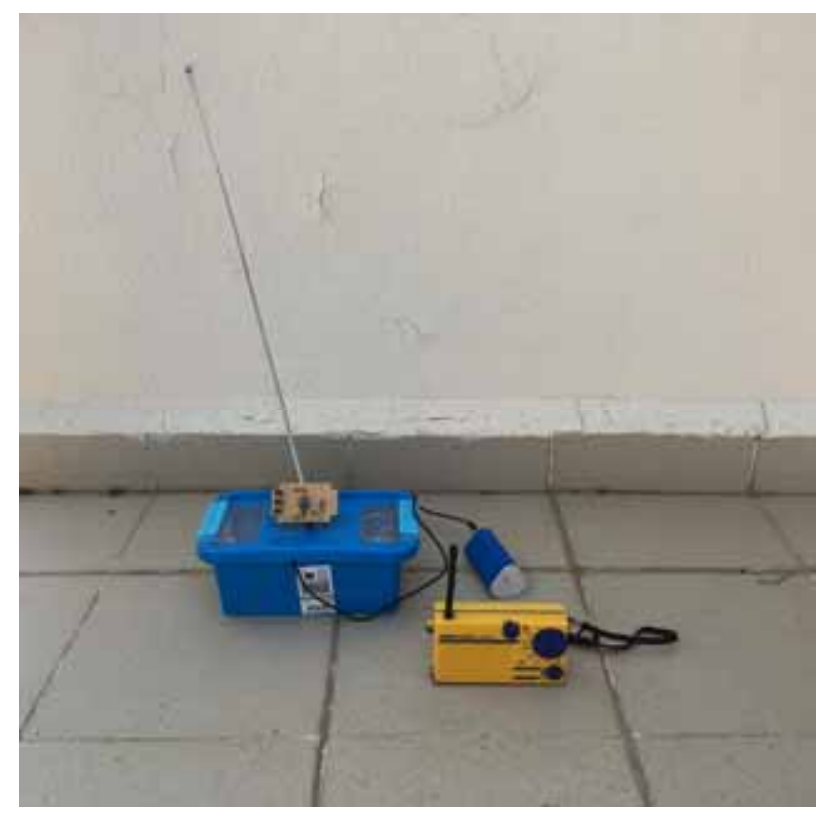

Figure 3: Workshop setup for Parasitic Radio

\section{REFERENCES}

Kahn, Douglas (2013) Earth Sound Earth Signal. Energies and Earth Magnitude in the Arts. University of California Press.

Vickery, Lindsey (2012) Adapting John Cage's Radio Music for digital performance Lindsay Vickery. Western Australian Academy of Performing Arts, Edith Cowan University

Howse, Martin. Gaffney, Nik. (2014) Approaching the Inexplicable. Medium. https://medium.com/aperiodicmesmerism/approaching-the-inexplicable7e1793b2a588

Morton, Timothy (2009). Ecology without Nature. Rethinking Environmental Aesthetics. Harvard

University Press.

Duarte Regino, Juan (2018) Aeolian Artefacts. Electronic Workshops in Computing. 